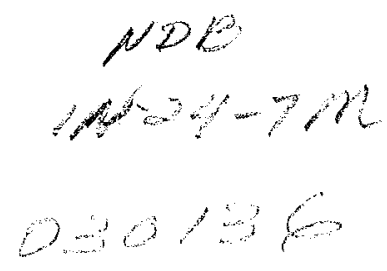

\title{
Two-Flux Green's Function Analysis for Transient Spectral Radiation in a Composite \\ R. Siegel
}

\section{Reprinted from}

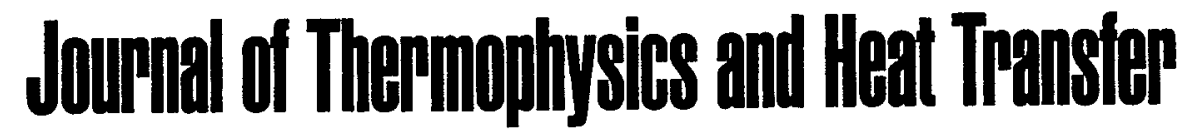

Volume 10, Number 4, Pages 681-688

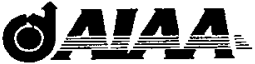

A publication of the

American Institute of Aeronautics and Astronautics, Inc.

1801 Alexander Bell Drive, Suite 500

Reston, VA 22091 


\title{
Two-Flux Green's Function Analysis for Transient Spectral Radiation in a Composite
}

\author{
Robert Siegel* \\ NASA Lewis Research Center, Cleveland, Ohio 44135
}

\begin{abstract}
An analysis is developed for obtaining transient temperatures in a two-layer semitransparent composite with spectrally dependent properties. Each external boundary of the composite is subjected to radiation and convection. The two-flux radiative transfer equations are solved by deriving a Green's function. This yields the local radiative heat source needed to numerically solve the transient energy equation. An advantage of the two-flux method is that isotropic scattering is included without added complexity. The layer refractive indices are larger than one. This produces internal reflections at the boundaries and the internal interface; the reflections are assumed diffuse. Spectral results using the Green's function method are verified by comparing with numerical solutions using the exact radiative transfer equations. Transient temperature distributions are given to illustrate the effect of radiative heating on one side of a composite with external convective cooling. The protection of a material from incident radiation is illustrated by adding scattering to the layer adjacent to the radiative source.
\end{abstract}

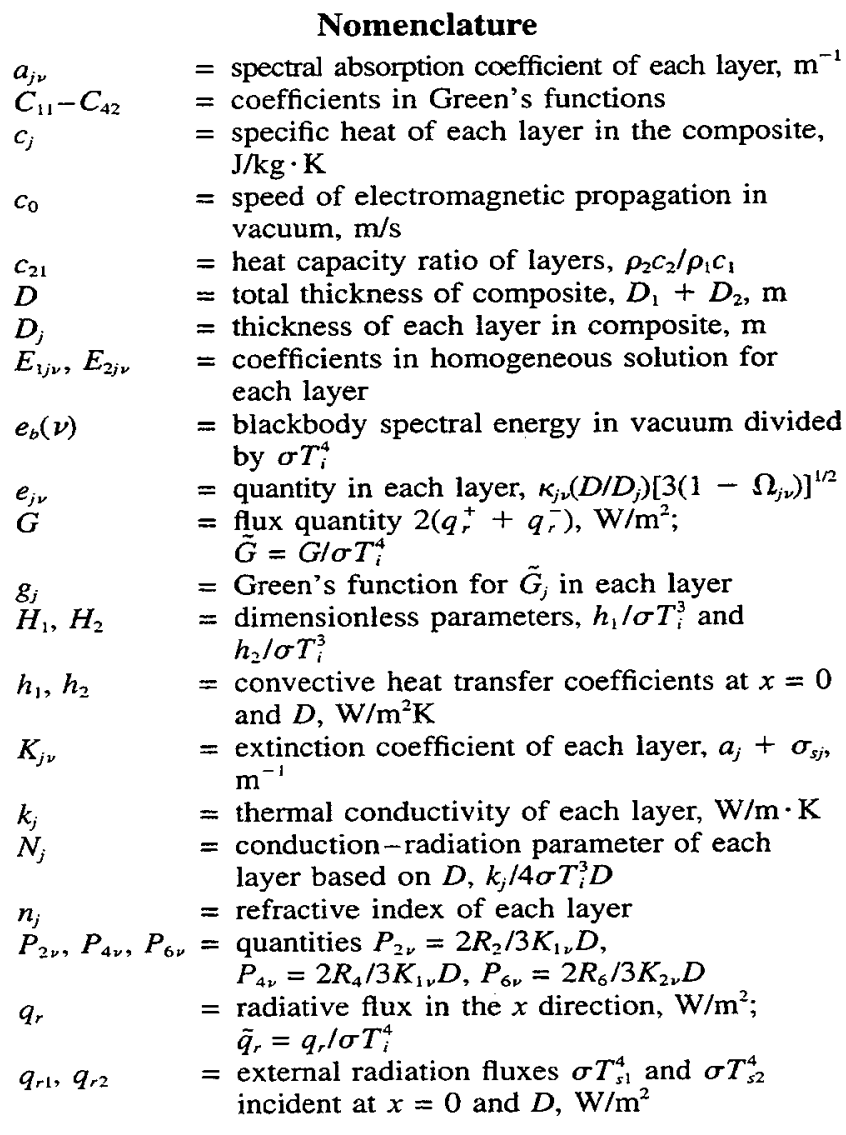

Received Feb. 19.1996; revision received June 11, 1996; accepted for publication June 17, 1996. Copyright (C) 1996 by the American Institute of Aeronautics and Astronautics, Inc. No copyright is asserted in the United States under Title 17, U.S. Code. The U.S. Government has a royalty-free license to exercise all rights under the copyright claimed herein for Governmental purposes. All other rights are reserved by the copyright owner.

* Senior Research Scientist, Research Academy, 21000 Brookpark Road. Fellow AIAA.
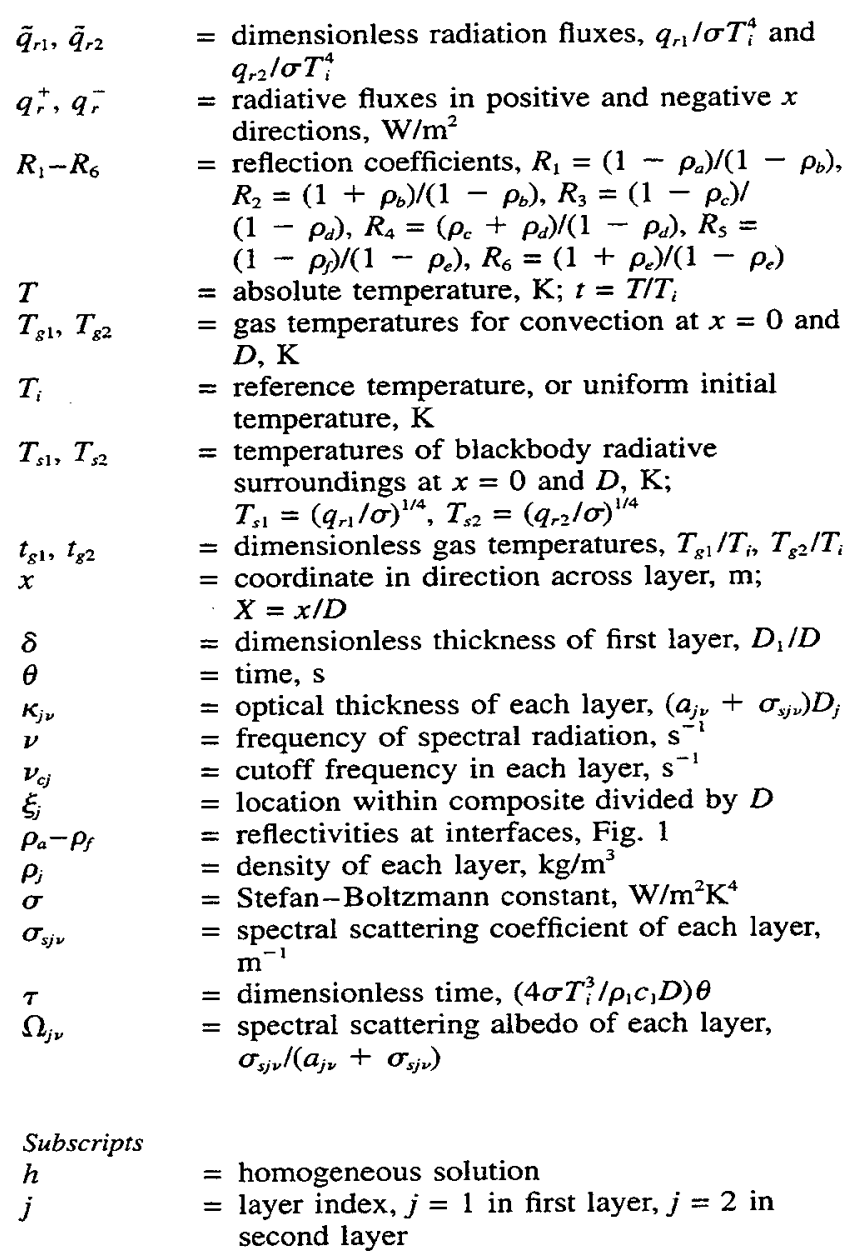

\section{Introduction}

D ETAILED transient temperatures are needed to examine heat transfer and thermal stress behavior for materials at high temperature that are partially transparent to thermal radiation. Applications include ceramic components, thermal 
protection coatings, tempering of glass windows, high-temperature insulation systems, and porous ceramic burners. For semitransparent materials where thermal radiation affects internal temperatures, transients have been studied much less than steady state.

For transient solutions, numerical procedures such as finite differences and finite elements have been used to solve the transient energy equation coupled with the radiative transfer relations. Some of the literature is reviewed in Refs. 1-7, and so a detailed review of transient solutions is not repeated here. A few investigations of particular interest for the present study provide analyses of composites with two or multiple layers. ${ }^{8-11}$ In Ref. 8 , the radiative transfer equations for absorbing layers with diffuse boundaries are solved numerically in combination with the transient energy equation; scattering is not included. A finite difference method is used in the spatial direction, and a Runge-Kutta method is used to move forward in time. A multilayered region with diffuse boundaries is considered in Ref. 9; scattering is not included. A finite difference method is used with a variable grid size. A two-layer composite is analyzed in Ref. 10 subjected to external radiation at one of its boundaries. Isotropic scattering is included, and the layers are gray. A Galerkin method is used to solve the radiative transfer relations, coupled with a finite difference solution of the transient energy equation.

The present analysis continues the development in Ref. 11 where a two-flux Green's function method was developed for a two-layer gray composite; spectral properties are now included. The two-flux approximation ${ }^{12}$ is used for the spectral radiative transfer equations. An advantage of the two-flux method is that isotropic scattering is included without additional effort, as compared with the need to solve the scattering integral equation at each time when using the exact transfer relations. For the general boundary conditions of external convection and radiation for a layer having diffuse interfaces, it was shown in Refs. 13 and 14 that the two-flux method provides accurate temperature distributions and heat fluxes. This was for steady state in single and composite layers with scattering included. For transient behavior, the two-flux method was shown to provide accurate results for a single layer, ${ }^{3}$ and for a two-layer gray composite. ${ }^{11}$

In the present analysis, the Green's function solution ${ }^{11}$ for the two-flux differential equations is extended for spectral property variations in a composite with general boundary conditions. This solution for the local radiative heat source is combined with an implicit finite difference solution of the transient energy equation using a tridiagonal matrix. Results are obtained to validate the numerical method and to illustrate some aspects of transient behavior. They compare, for example, gray and spectral calculations, and show how additional scattering in one layer of a composite can alter the temperature distribution in an adjacent layer. The solution can be applied to examine many transient effects in a two-layer composite with spectral properties.

\section{Analysis}

The analysis is for a plane two-layer composite, Fig. 1, that has different spectral radiative absorption and scattering properties in each layer. The layers are semitransparent absorbing dielectrics with refractive indices larger than one. There is heat conduction and isotropic scattering in each layer. The external boundaries and the interface between the layers are assumed diffuse. The diffuse surface reflectivities are determined from the $n_{j}$ using the Fresnel equations; since the $n_{j}$ are assumed independent of $\nu$, the reflectivities do not depend on frequency. Initially, the composite has a temperature distribution $T(x, 0)$, and $T_{i}$ is a characteristic temperature of $T(x, 0)$ used for dimensionless quantities. To begin a transient, the composite is subjected to surroundings so that each boundary can receive or lose radiant energy, and is in contact with a convecting gas. Transient temperature distributions are obtained until steady

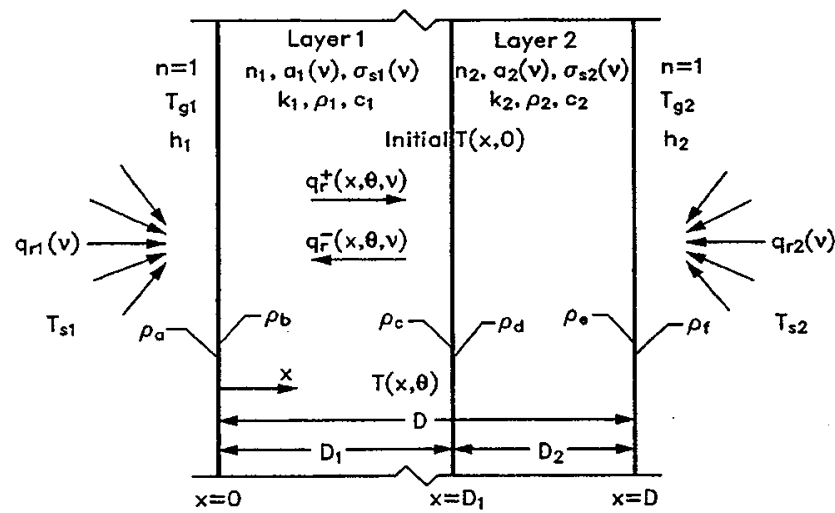

Fig. 1 Geometry and nomenclature for transient radiation and conduction in a two-layer semitransparent composite with spectrally dependent properties and isotropic scattering.

state is reached, corresponding to the imposed external conditions.

Energy Equation and Two-Flux Relations

for a Two-Layer Composite

The dimensionless energy equation for transient temperatures in each layer is ${ }^{3}$

$$
\begin{array}{cc}
\frac{\partial t(X, \tau)}{\partial \tau}=N_{1} \frac{\partial^{2} t(X, \tau)}{\partial X^{2}}-\frac{1}{4} \frac{\partial \tilde{q}_{r}(X, \tau)}{\partial X} & 0 \leq X<\delta \\
\frac{\partial t(X, \tau)}{\partial \tau}=\frac{N_{2}}{c_{21}} \frac{\partial^{2} t(X, \tau)}{\partial X^{2}}-\frac{1}{4 c_{21}} \frac{\partial \tilde{q}_{r}(X, \tau)}{\partial X} & \delta<X \leq 1
\end{array}
$$

The last term on the right in each equation is the local heat source, including all frequencies, produced by radiative absorption, emission, and scattering. Properties are assumed independent of temperature. The $\partial \tilde{q}_{r}(X, \tau) / \partial X$ is obtained in terms of the instantaneous temperature distribution by using the two-flux relations; these have provided accurate results in previous steady-state ${ }^{13.14}$ and transient $\mathrm{t}^{3.11}$ analyses. With $n_{1}$ and $n_{2}$ assumed independent of frequency, the spectral two-flux equations using the Milne-Eddington approximation are $^{12}$

$$
\begin{aligned}
& \frac{\partial \tilde{q}_{r}(X, \tau, \nu)}{\partial X}=\frac{\kappa_{1 \nu}}{\delta}\left(1-\Omega_{1 \nu}\right)\left[4 n_{1}^{2} e_{b}(X, \tau, \nu)\right. \\
& \left.\quad-\tilde{G}_{1}(X, \tau, \nu)\right] \quad 0 \leq X<\delta \\
& \frac{\partial \tilde{q}_{r}(X, \tau, \nu)}{\partial X}=\frac{\kappa_{2 \nu}}{1-\delta}\left(1-\Omega_{2 \nu}\right)\left[4 n_{2}^{2} e_{b}(X, \tau, \nu)\right. \\
& \left.-\tilde{G}_{2}(X, \tau, \nu)\right] \quad \delta<X \leq 1
\end{aligned}
$$

where $\tilde{G}_{1}(X, \tau, \nu)$ and $\tilde{G}_{2}(X, \tau, \nu)$ are related to $\tilde{q}_{r}(X, \tau, \nu)$ by

$$
\begin{array}{rr}
\frac{\partial \tilde{G}_{1}(X, \tau, \nu)}{\partial X}=-3 \frac{\kappa_{1 \nu}}{\delta} \tilde{q}_{r}(X, \tau, \nu) & 0 \leq X<\delta \\
\frac{\partial \tilde{G}_{2}(X, \tau, \nu)}{\partial X}=-3 \frac{\kappa_{2 \nu}}{1-\delta} \tilde{q}_{r}(X, \tau, \nu) & \delta<X \leq 1
\end{array}
$$

The $\tilde{q}_{r}$ and $\tilde{G}$ are related to the positive and negative radiative fluxes in Fig. 1 by $\tilde{q}_{r}=\tilde{q}_{r}^{+}-\tilde{q}_{r}^{-}$and $\tilde{G}=2\left(\tilde{q}_{r}^{+}+\tilde{q}_{r}^{-}\right)$.

\section{Boundary, Interface, and Initial Conditions}

For a semitransparent material, radiation is absorbed within the material interior and not at a surface, since the surface has no thickness. Hence, the relations between conduction and convection at the boundaries do not include radiative terms

$$
\left.\frac{\partial t(X, \tau)}{\partial X}\right|_{x=0}=-\frac{H_{1}}{4 N_{1}}\left[t_{s 1}-t(0, \tau)\right]
$$




$$
\left.\frac{\partial t(X, \tau)}{\partial X}\right|_{X=1}=-\frac{H_{2}}{4 N_{2}}\left[t(1, \tau)-t_{g 2}\right]
$$

At the internal interface, $X=\delta$, there is continuity of temperature and heat conduction

$$
\begin{gathered}
t(\delta-, \tau)=t(\delta+, \tau) \\
\left.N_{1} \frac{\partial t(X, \tau)}{\partial X}\right|_{X=\delta^{-}}=\left.N_{2} \frac{\partial t(X, \tau)}{\partial X}\right|_{X=\delta+}
\end{gathered}
$$

The boundary conditions for radiation must include the effects of external and internal reflections at the outer surfaces that are exposed to spectral fluxes $q_{r 1}(\nu)$ and $q_{r 2}(\nu)$. The conditions have been developed by considering the incident, reflected, and transmitted fluxes at a boundary ${ }^{13}$

$$
\begin{gathered}
\tilde{G}_{1}(0, \tau, \nu)=4 R_{1} \tilde{q}_{r 1}(\nu)+\left.\frac{2 \delta}{3 \kappa_{1 \nu}} R_{2} \frac{\partial \tilde{G}_{1}(X, \tau, \nu)}{\partial X}\right|_{X=0} \\
\tilde{G}_{2}(1, \tau, \nu)=4 R_{5} \tilde{q}_{r 2}(\nu)-\left.\frac{2(1-\delta)}{3 \kappa_{2 \nu}} R_{6} \frac{\partial \tilde{G}_{2}(X, \tau, \nu)}{\partial X}\right|_{X=1}
\end{gathered}
$$

At the interface between the two layers the relation between reflected and transmitted fluxes gives ${ }^{14}$

$$
\tilde{G}_{2}(\delta, \tau, \nu)=R_{3} \tilde{G}_{1}(\delta, \tau, \nu)+\left.\frac{2 \delta}{3 \kappa_{1 \nu}} R_{4} \frac{\partial \tilde{G}_{1}(\delta, \tau, \nu)}{\partial X}\right|_{X=\delta}
$$

There is a discontinuity in $\tilde{G}$ at an interface, so that $\tilde{G}_{2}(\delta, \tau$, $\nu) \neq \tilde{G}_{1}(\delta, \tau, \nu)$. The radiative flux, however, is continuous at the internal interface, and so from Eq. (3)

$$
\left.\frac{\delta}{\kappa_{1 \nu}} \frac{\partial \tilde{G}_{1}(X, \tau, \nu)}{\partial X}\right|_{X=\delta}=\left.\frac{1-\delta}{\kappa_{2 \nu}} \frac{\partial \tilde{G}_{2}(X, \tau, \nu)}{\partial X}\right|_{X=\delta}
$$

\section{Green's Function to Determine $\tilde{G}(X, \tau, \nu)$}

\section{in a Two-Layer Composite}

To find $\partial \tilde{q}_{r}(X, \tau) / \partial X$ for Eq. (1), the $\tilde{G}_{j}(X, \tau, \nu)$ are needed for use in Eq. (2), which is then integrated over all $\nu$. Green's functions are developed to obtain $\bar{G}_{j}(X, \tau, \nu)$ in terms of $e_{b}(X$, $\tau, \nu)$. Equations (3a) and (3b) are differentiated in $X$, and $\partial \tilde{q}_{r}(X, \tau, \nu) / \partial X$ is then eliminated from Eqs. (2a) and (2b). The resulting differential equation for $\widetilde{G}_{j}(X, \tau, \nu)$ is

$$
\begin{aligned}
& \frac{\partial^{2} \tilde{G}_{j}(X, \tau, \nu)}{\partial X^{2}}-e_{j \nu}^{2} \tilde{G}_{j}(X, \tau, \nu)=-4 e_{j \nu}^{2} n_{j}^{2} e_{b}(X, \tau, \nu) \\
& j=1,2
\end{aligned}
$$

For convenience, the $\tau$ functional notation is omitted in the following derivation. The homogeneous part of Eq. (7) is satisfied by $\cosh \left(e_{j \nu} X\right)$ and $\sinh \left(e_{j \nu} X\right)$, and so the Green's functions have the form ${ }^{15}$

$$
\begin{aligned}
& g_{1}\left(X, \xi_{1}, \nu\right) \\
& = \begin{cases}C_{11}\left(\xi_{1}, \nu\right) \cosh e_{1 \nu} X+C_{21}\left(\xi_{1}, \nu\right) \sinh e_{1 \nu} X & 0 \leq X<\xi_{1} \\
C_{31}\left(\xi_{1}, \nu\right) \cosh e_{1 \nu} X+C_{41}\left(\xi_{1}, \nu\right) \sinh e_{1 \nu} X & \xi_{1}<X \leq \delta\end{cases}
\end{aligned}
$$

$$
\begin{aligned}
& g_{2}\left(X, \xi_{2}, \nu\right) \\
& = \begin{cases}C_{12}\left(\xi_{2}, \nu\right) \cosh e_{2 \nu} X+C_{22}\left(\xi_{2}, \nu\right) \sinh e_{2 \nu} X & \delta \leq X<\xi_{2} \\
C_{32}\left(\xi_{2}, \nu\right) \cosh e_{2 \nu} X+C_{42}\left(\xi_{2}, \nu\right) \sinh e_{2 \nu} X & \xi_{2}<X \leq 1\end{cases}
\end{aligned}
$$

To obtain the coefficients $C_{1 j}\left(\xi_{j}, \nu\right)$ to $C_{4 j}\left(\xi_{j}, \nu\right)$, the $g_{j}\left(X, \xi_{j}\right.$, $\nu)$ in Eq. (8) must satisfy the following relations obtained from the homogeneous forms of Eqs. (5a) and (5b):

$$
\begin{gathered}
g_{1}(0, \nu)-\left.\frac{2 \delta}{3 \kappa_{1 \nu}} R_{2} \frac{\partial g_{1}(X, \nu)}{\partial X}\right|_{X=0}=0 \\
g_{2}(1, \nu)+\left.\frac{2(1-\delta)}{3 \kappa_{2 \nu}} R_{6} \frac{\partial g_{2}(X, \nu)}{\partial X}\right|_{X=1}=0
\end{gathered}
$$

By using Eqs. (8a) and (8b) in Eqs. (9a) and (9b) to evaluate $C_{1 j}(\xi, \nu)$ to $C_{4 j}(\xi, \nu)$, the $g_{j}(X, \xi, \nu)$ reduce to

$$
\begin{aligned}
& g_{1}\left(X, \xi_{1}, \nu\right) \\
& = \begin{cases}C_{21}\left(\xi_{1}, \nu\right)\left(\sinh e_{1 \nu} X+P_{2 \nu} e_{1 \nu} \cosh e_{1 \nu} X\right) & 0 \leq X<\xi_{1} \\
C_{41}\left(\xi_{1}, \nu\right)\left(\sinh e_{1 \nu} X-F_{1 \nu} \cosh e_{1 \nu} X\right) & \xi_{1}<X \leq \delta \\
(10 \mathrm{a})\end{cases} \\
& g_{2}\left(X, \xi_{2}, \nu\right) \\
& = \begin{cases}C_{22}\left(\xi_{2}, \nu\right)\left(\sinh e_{2 \nu} X-F_{2 \nu} \cosh e_{2 \nu} X\right) & \delta \leq X<\xi_{2} \\
C_{42}\left(\xi_{2}, \nu\right)\left(\sinh e_{2 \nu} X-F_{3 \nu} \cosh e_{2 \nu} X\right) & \xi_{2}<X \leq 1\end{cases}
\end{aligned}
$$

where $F_{1 \nu} \equiv\left(R_{3} \sinh e_{1 \nu} \delta+P_{4 \nu} e_{1 \nu} \cosh e_{1 \nu} \delta\right) /\left(R_{3} \cosh e_{1 \nu} \delta+\right.$ $P_{4 \nu} e_{1 \nu} \sinh e_{1 \nu} \delta$ ), $F_{2 \nu} \equiv \cosh e_{2 \nu} \delta / \sinh e_{2 \nu} \delta$, and $F_{3 \nu} \equiv$ (sinh $\left.e_{2 \nu}+P_{5 \nu} e_{2 \nu} \cosh e_{2 \nu}\right) /\left(\cosh e_{2 \nu}+P_{6 \nu} e_{2 \nu} \sinh e_{2 \nu}\right)$.

The $C_{2 j}(\xi, \nu)$ and $C_{4 j}\left(\xi_{j}, \nu\right)$ in Eq. (10) are obtained by applying the two conditions ${ }^{15}$ that $g_{j}\left(X, \xi_{j}, \nu\right)$ is continuous at $X=\xi_{j}$, and that for increasing $X$, the $\partial g_{j}\left(X, \xi_{j}, \nu\right) / \partial X$ passes through a discontinuity of -1 at each $X=\xi_{j}$. This yields the relations for $C_{21}\left(\xi_{1}, \nu\right)$ and $C_{41}\left(\xi_{1}, \nu\right)$ :

$$
\begin{aligned}
& C_{21}\left(\xi_{1}, \nu\right)\left(\sinh e_{1 \nu} \xi_{1}+P_{2 \nu} e_{1 \nu} \cosh e_{1 \nu} \xi_{1}\right) \\
& \quad=C_{41}\left(\xi_{1}, \nu\right)\left(\sinh e_{1 \nu} \xi_{1}-F_{1 \nu} \cosh e_{1 \nu} \xi_{1}\right) \\
& C_{41}\left(\xi_{1}, \nu\right) e_{1 \nu}\left[\cosh e_{1 \nu} \xi_{1}-F_{1 \nu} \sinh e_{1 \nu} \xi_{1}\right) \\
& \quad-C_{21}\left(\xi_{1}, \nu\right) e_{1 \nu}\left(\cosh e_{1 \nu} \xi_{1}+P_{2 \nu} e_{1 \nu} \sinh e_{1 \nu} \xi_{1}\right)=-1
\end{aligned}
$$

and for $C_{22}\left(\xi_{2}, \nu\right)$ and $C_{42}\left(\xi_{2}, \nu\right)$ :

$$
\begin{aligned}
& C_{22}\left(\xi_{2}, \nu\right)\left(\sinh e_{2 \nu} \xi_{2}-F_{2 \nu} \cosh e_{2 \nu} \xi_{2}\right) \\
& \quad=C_{42}\left(\xi_{2}, \nu\right)\left(\sinh e_{2 \nu} \xi_{2}-F_{3 \nu} \cosh e_{2 \nu} \xi_{2}\right) \\
& C_{42}\left(\xi_{2}, \nu\right) e_{2 \nu}\left(\cosh e_{2 \nu} \xi_{2}-F_{3 \nu} \sinh e_{2 \nu} \xi_{2}\right) \\
& \quad-C_{22}\left(\xi_{2}, \nu\right) e_{2 \nu}\left(\cosh e_{2 \nu} \xi_{2}-F_{2 \nu} \sinh e_{2 \nu} \xi_{2}\right)=-1
\end{aligned}
$$

Equations (11a) and (11b) are solved for $C_{21}\left(\xi_{1}, \nu\right)$ and $C_{41}\left(\xi_{1}\right.$, $\nu)$, and Eqs. (12a) and (12b) for $C_{22}\left(\xi_{2}, \nu\right)$ and $C_{42}\left(\xi_{2}, \nu\right)$. After substituting into Eqs. (10a) and (10b), the final Green's functions are

$$
g_{1}\left(X, \xi_{1}, \nu\right)= \begin{cases}{\left[\frac{\left(\tanh e_{1 \nu} \xi_{1}+P_{2 \nu} e_{1 \nu}\right) \cosh e_{1 \nu} \xi_{1}}{\operatorname{Denom}_{1 \nu}}\right]\left\{R_{3} \tanh \left[e_{1 \nu}(\delta-X x)\right]+P_{4 \nu} e_{1 \nu}\right\} \cosh \left[e_{1 \nu}(\delta-X)\right]} & 0 \leq X<\xi_{1} \\ {\left[\frac{\left\{R_{3} \tanh \left[e_{1 \nu}\left(\delta-\xi_{1}\right)\right]+P_{4 \nu} e_{1 \nu}\right\} \cosh \left[e_{1 \nu}\left(\delta-\xi_{1}\right)\right]}{\operatorname{Denom}_{1 \nu}}\right]\left[\left(\tanh e_{1 \nu} X+P_{2 \nu} e_{1 \nu}\right) \cosh e_{1 \nu} X\right]} & \xi_{1}<X \leq \delta\end{cases}
$$




$$
g_{2}\left(X, \xi_{2}, \nu\right)= \begin{cases}{\left[\frac{\cosh \left[e_{2 \nu}\left(\xi_{2}-\delta\right)\right]}{\operatorname{Denom}_{2 \nu}}\right]\left\{\tanh \left[e_{2 \nu}(1-X)\right]+P_{6 \nu} e_{2 \nu}\right\} \cosh \left[e_{2 \nu}(1-X)\right]} & \delta \leq X<\xi_{2} \\ {\left[\frac{\left\{\tanh \left[e_{2 \nu}\left(1-\xi_{2}\right)\right]+P_{6 \nu} e_{2 \nu}\right\} \cosh \left[e_{2 \nu}\left(1-\xi_{2}\right)\right]}{\operatorname{Denom}_{2 \nu}}\right] \cosh \left[e_{2 \nu}(X-\delta)\right]} & \xi_{2}<X \leq 1\end{cases}
$$

where

$$
\begin{aligned}
& \operatorname{Denom}_{1 \nu}=e_{1 \nu}\left\{\left[\left(R_{3}+P_{2 \nu} P_{4 \nu} e_{1 \nu}^{2}\right) \tanh e_{1 \nu} \delta\right.\right. \\
& \left.\left.\quad+e_{1 \nu}\left(P_{4 \nu}+P_{2 \nu} R_{3}\right)\right] \cosh e_{1 \nu} \delta\right\} \\
& \operatorname{Denom}_{2 \nu}=e_{2 \nu}\left\{1+e_{2 \nu} P_{6 \nu} \tanh \left[e_{2 \nu}(1-\delta)\right]\right\} \\
& \quad \times \cosh \left[e_{2 \nu}(1-\delta)\right]
\end{aligned}
$$

The $g_{j}\left(X, \xi_{j}, \nu\right)$ in Eq. (13) are used to account for the nonhomogeneous term containing $e_{b}(X, \tau, \nu)$ in Eq. (7) when computing $\tilde{G}_{j}(X, \tau, \nu)$ at each time during the transient. To obtain the complete solution for $\tilde{G}_{j}(X, \tau, \nu)$, the homogeneous solution is needed for Eq. (7). This has the form $\widetilde{G}_{h j}(X, \nu)=$ $E_{1 j \nu} \sinh e_{j \nu} X+E_{2 j \nu} \cosh e_{j \nu} X$. The boundary conditions in Eqs. (5) and (6) are applied and the result is four simultaneous equations for the $E_{1 j \nu}$ and $E_{2 j \nu}$ coefficients:

$$
\begin{gathered}
P_{2 \nu} e_{1 \nu} E_{11 \nu}-E_{21 \nu}=-4 R_{1} \tilde{q}_{r 1}(\nu) \\
\left(\frac{e_{1 \nu} \delta}{\kappa_{1 \nu}} \cosh e_{1 \nu} \delta\right) E_{11 \nu}+\left(\frac{e_{1 \nu} \delta}{\kappa_{1 \nu}} \sinh e_{1 \nu} \delta\right) E_{21 \nu} \\
-\left[\frac{e_{2 \nu}(1-\delta)}{\kappa_{2 \nu}} \cosh e_{2 \nu} \delta\right] E_{12 \nu} \\
-\left[\frac{e_{2 \nu}(1-\delta)}{\kappa_{2 \nu}} \sinh e_{2 \nu} \delta\right] E_{22 \nu}=-C_{1 \nu}
\end{gathered}
$$

$\left(R_{3} \sinh e_{1 \nu} \delta+P_{4 \nu} e_{1 \nu} \cosh e_{1 \nu} \delta\right) E_{11 \nu}$

$+\left(R_{3} \cosh e_{1 \nu} \delta+P_{4 \nu} e_{1 \nu} \sinh e_{1 \nu} \delta\right) E_{21 \nu}$

$-\left(\sinh e_{2 \nu} \delta\right) E_{12 \nu}-\left(\cosh e_{2 \nu} \delta\right) E_{22 \nu}=C_{2 \nu}$

$\left(\sinh e_{2 \nu}+P_{6 \nu} e_{2 \nu} \cosh e_{2 \nu}\right) E_{12 \nu}$

$$
+\left(\cosh e_{2 \nu}+P_{6 \nu} e_{2 \nu} \sinh e_{2 \nu}\right) E_{22 \nu}=4 R_{5} \tilde{q}_{r 2}(\nu)
$$

where by using the Green's functions

$$
\begin{aligned}
C_{1 \nu} & =-4 R_{3} e_{1 \nu}^{3} n_{1}^{2} \\
& \times \frac{\delta}{\kappa_{1 \nu}} \int_{0}^{\delta} \frac{\left(\tanh e_{1 \nu} \xi_{1}+P_{2 \nu} e_{1 \nu}\right) \cosh e_{1 \nu} \xi_{1}}{\operatorname{Denom}_{1 \nu}} e_{b}\left(\xi_{1}, \nu\right) \mathrm{d} \xi_{1} \\
C_{2 \nu} & =4 e_{2 \nu}^{2} n_{2}^{2} \\
& \times \int_{\delta}^{1} \frac{\left\{\tanh \left[e_{2 \nu}\left(1-\xi_{2}\right)\right]+P_{6 \nu} e_{2 \nu}\right\} \cosh \left[e_{2 \nu}\left(1-\xi_{2}\right)\right]}{\operatorname{Denom}_{2 \nu}} \\
& \times e_{b}\left(\xi_{2}, \nu\right) \mathrm{d} \xi_{2}
\end{aligned}
$$

By adding $\tilde{G}_{h j}(X, \nu)$ to the nonhomogeneous solutions obtained by using $g_{j}(X, \xi, \nu)$, the general spectral solution of Eq. (7) in each layer at each time is

$$
\begin{aligned}
& \tilde{G}_{1}(X, \tau, \nu)=\tilde{G}_{h 1}(X, \tau, \nu) \\
& \quad+4 e_{1 \nu}^{2} n_{1}^{2} \int_{0}^{\delta} g_{1}\left(X, \tau, \xi_{1}, \nu\right) e_{b}\left(\xi_{1}, \tau, \nu\right) \mathrm{d} \xi_{1}
\end{aligned}
$$

$$
\begin{aligned}
& \tilde{G}_{2}(X, \tau, \nu)=\tilde{G}_{h 2}(X, \tau, \nu) \\
& \quad+4 e_{2 \nu}^{2} n_{2}^{2} \int_{\delta}^{1} g_{2}\left(X, \tau, \xi_{2}, \nu\right) e_{b}\left(\xi_{2}, \tau, \nu\right) \mathrm{d} \xi_{2}
\end{aligned}
$$

The forms for two spectral bands can be written in the same way as in Ref. 3, where a spectral Green's function analysis is given for a single layer.

\section{Numerical Solution}

Starting with a specified $t(X, 0)$, the blackbody function $e_{b}(X$, $0, \nu)$ is known. The Green's functions are used in Eqs. (15a) and $(15 b)$ to obtain $\tilde{G}_{1}(X, 0, \nu)$ and $\tilde{G}_{2}(X, 0, \nu)$, and the $\partial$ $\tilde{q}_{r}(X, 0, \nu) / \partial X$ is then evaluated from Eq. (2). This spectral quantity is integrated over all $\nu$, so that it can be used in the energy equation that involves total energy quantities (the results given later are for two spectral bands with the same cutoff frequency in both layers). Using Eq. (1), $t(X, \tau)$ is integrated forward one time increment using the finite difference relations that follow. Then the new temperature distribution is used with the Green's functions to obtain $\tilde{G}_{j}(X, \Delta \tau, \nu)$ and $\partial \tilde{q}_{r}(X, \Delta \tau, \nu) /$ $\partial X$. The $\partial \bar{q}_{r}(X, \Delta \tau, \nu) / \partial X$ is integrated over all $\nu$ and is used in Eq. (1) to continue on to obtain $t(X, \tau)$ at the next time step.

From Eqs. (1a) and (1b), the following implicit finite difference algorithms are used at the interior points of a uniform grid to advance the temperature distributions each $\Delta \tau$ in each layer:

$$
\begin{aligned}
& -\frac{N_{1} \Delta \tau}{(\Delta X)^{2}} t(X-\Delta X, \tau+\Delta \tau)+\left[1+\frac{2 N_{1} \Delta \tau}{(\Delta X)^{2}}\right] t(X, \tau+\Delta \tau) \\
& -\frac{N_{1} \Delta \tau}{(\Delta X)^{2}} t(X+\Delta X, \tau+\Delta \tau)=t(X, \tau)-\left.\frac{\Delta \tau}{4} \frac{\partial \bar{q}_{r}}{\partial X}\right|_{x, \tau} \\
& \quad 0<X<\delta \\
& -\frac{N_{2} \Delta \tau}{c_{21}(\Delta X)^{2}} t(X-\Delta X, \tau+\Delta \tau)+\left[1+\frac{2 N_{2} \Delta \tau}{c_{21}(\Delta X)^{2}}\right] t(X, \tau+\Delta \tau) \\
& -\frac{N_{2} \Delta \tau}{c_{21}(\Delta X)^{2}} t(X+\Delta X, \tau+\Delta \tau)=t(X, \tau)-\left.\frac{\Delta \tau}{4 c_{21}} \frac{\partial \tilde{q}_{r}}{\partial X}\right|_{x . \tau} \\
& \delta<X<1
\end{aligned}
$$

For the boundary points $X=0$ and 1 , Eq. (16) is applied, and the $t(-\Delta X, \tau+\Delta \tau)$ and $t(1+\Delta X, \tau+\Delta \tau)$ that appear are eliminated by using the boundary conditions Eqs. (4a) and (4b), expressed as

$$
\begin{aligned}
& \frac{t(\Delta X, \tau+\Delta \tau)-t(-\Delta X, \tau+\Delta \tau)}{2 \Delta X} \\
& =-\frac{H_{1}}{4 N_{1}}\left[t_{\varepsilon 1}-t(0, \tau+\Delta \tau)\right] \\
& \frac{t(1+\Delta X, \tau+\Delta \tau)-t(1-\Delta X, \tau+\Delta \tau)}{2 \Delta X} \\
& =-\frac{H_{2}}{4 N_{2}}\left[t(1, \tau+\Delta \tau)-t_{g 2}\right]
\end{aligned}
$$


This yields the finite difference relations at $X=0$ and 1 as

$$
\begin{aligned}
& {\left[1+\frac{2 N_{1} \Delta \tau}{(\Delta X)^{2}}+\frac{H_{1} \Delta \tau}{2 \Delta X}\right] t(0, \tau+\Delta \tau)} \\
& -\frac{2 N_{1} \Delta \tau}{(\Delta X)^{2}} t(\Delta X, \tau+\Delta \tau)=t(0, \tau)-\left.\frac{\Delta \tau}{4} \frac{\partial \tilde{q}_{r}}{\partial X}\right|_{0 . \tau}+\frac{H_{1} \Delta \tau}{2 \Delta X} t_{g 1} \\
& -\frac{2 N_{2} \Delta \tau}{c_{21}(\Delta X)^{2}} t(1-\Delta X, \tau+\Delta \tau)+\left[1+\frac{2 N_{2} \Delta \tau}{c_{21}(\Delta X)^{2}}+\frac{H_{2} \Delta \tau}{2 c_{21} \Delta X}\right] \\
& \quad \times t(1, \tau+\Delta \tau)=t(1, \tau)-\left.\frac{\Delta \tau}{4 c_{21}} \frac{\partial \tilde{q}_{r}}{\partial X}\right|_{1, \tau}+\frac{H_{2} \Delta \tau}{2 c_{21} \Delta X} t_{g 2}
\end{aligned}
$$

The condition at the internal interface, Eq. (4d), is written by using three-point derivatives, and Eq. (4c) is substituted to give

$$
\begin{aligned}
& \frac{N_{1}}{2 \Delta X}[t(\delta-2 \Delta X, \tau)-4 t(\delta-\Delta X, \tau)+3 t(\delta, \tau)] \\
& \quad=\frac{N_{2}}{2 \Delta X}[-t(\delta+2 \Delta X, \tau)+4 t(\delta+\Delta X, \tau)-3 t(\delta, \tau)]
\end{aligned}
$$

Equation (16) is written for $t$ at $X+\Delta X$ and $X-\Delta X$, and these relations are used to eliminate the nontridiagonal elements $t(\delta-2 \Delta X, \tau)$ and $t(\delta+2 \Delta X, \tau)$. The result is a tridiagonal finite difference expression at the internal interface

$$
\begin{aligned}
& {\left[\frac{(\Delta X)^{2}}{\Delta \tau}-2 N_{1}\right] t(\delta-\Delta X, \tau+\Delta \tau)+2\left(N_{1}+N_{2}\right) t(\delta, \tau+\Delta \tau)} \\
& \quad+\left[\frac{c_{21}(\Delta X)^{2}}{\Delta \tau}-2 N_{2}\right] t(\delta+\Delta X, \tau+\Delta \tau) \\
& \quad=\frac{(\Delta X)^{2}}{\Delta \tau}\left[t(\delta-\Delta X, \tau)+c_{21} t(\delta+\Delta X, \tau)\right] \\
& \quad-\frac{(\Delta X)^{2}}{4}\left(\left.\frac{\partial \tilde{q}_{r}}{\partial X}\right|_{\delta-\Delta X . \tau}+\left.\frac{\partial \tilde{q}_{r}}{\partial X}\right|_{\delta+\Delta X . \tau}\right)
\end{aligned}
$$

The $t(X, \tau)$ is advanced to the next $\Delta \tau$ using Eqs. (16-18) solved as a tridiagonal matrix using the algorithm in Ref. 16. As described in Ref. 4, overall energy balances were checked at each time step by using the calculated temperature distributions to evaluate the amounts contributed by radiation, convection, and internal energy storage. For the grid sizes used, the overall energy balance was satisfied within $0.5 \%$ throughout the transient calculations. After checking various grid sizes, it was found that 81 evenly spaced points across each layer gave accurate grid-independent solutions for $\kappa_{j \nu}<3$ in each layer. For $\kappa_{j \nu}>3,101$ points were used, which gave accurate solutions for $\kappa_{j y} \leq 10$. A time increment of $\Delta \tau=$ 0.0025 provided stable and time-accurate solutions for the results calculated here, as validated by comparisons with some previous numerical solutions using the exact radiative transfer relations.

\section{Results and Discussion}

The objective of the analysis was to develop a convenient and accurate method for obtaining transient temperature distributions in a two-layer semitransparent composite with spectral radiation properties, isotropic scattering, and differing refractive indices in each layer. The composite is subjected to external convection and radiation on both sides. A Green's function solution was derived for the spectral two-flux radiative transfer equations in two layers, and for the results given here, the solution was applied in two spectral bands with the same cutoff frequency in both layers. By summing over the two bands, the Green's function is used to obtain the radiative energy source term in the transient energy equation at each time step. The energy equation was solved with an implicit finite difference method that includes convective boundary conditions, and the required temperature and heat conduction conditions at the internal interface. Some of the transient results that will be presented are to verify the method for spectral calculations. This is done by comparing transient temperatures with previous spectral steady-state and transient results obtained or verified with the exact radiative transfer equations. The other figures that follow illustrate the variety of transient results with spectral effects that can be obtained with the present combined analytical and computational procedure.

The transient temperatures given here begin from a uniform temperature distribution $t(X, 0)=1$, although the solution applies for a general distribution $t(X, 0)$. For a partial validation of the present method, the solid lines in Fig. 2 are transient results for a two-band symmetric case using the same parameters as the transient solution in Fig. 6c of Ref. 3. The twoband spectral optical thicknesses of the layers in order of increasing frequency are $\kappa_{1 \nu}=\kappa_{2 \nu}=5$ and 0.05 . For $\tau>0$, the composite is exposed on both sides to a high-temperature gas at $T_{g 1}=T_{g 2}=4 T_{i}$, and there is large convective heating on both sides. The surroundings remain at $T_{s 1}=T_{s 2}=T_{i}$, so that as the temperatures rise, radiative cooling occurs from within the composite interior. Radiative cooling has a strong effect, and the transient interior temperatures are always considerably below the surface temperatures. At steady state, the interior temperatures in the central region of the composite are close to the uniform temperature for a composite that is opaque. The present results duplicated those in Ref. 3, where a single spectral layer was analyzed, and additional calculations were then made to illustrate the effect of heat capacity in the second layer of a two-layer composite. The dashed lines in Fig. 2 illustrate how increased heat capacity can delay the transient response. Heat capacity, however, does not alter the steady-state temperature distribution.

Figure 3 provides a comparison with a numerical solution of the exact transfer equations for steady state given in Fig. 3 of Ref. 14. The transient results at large time agree very well with this steady-state solution, which is for a two-layer com-

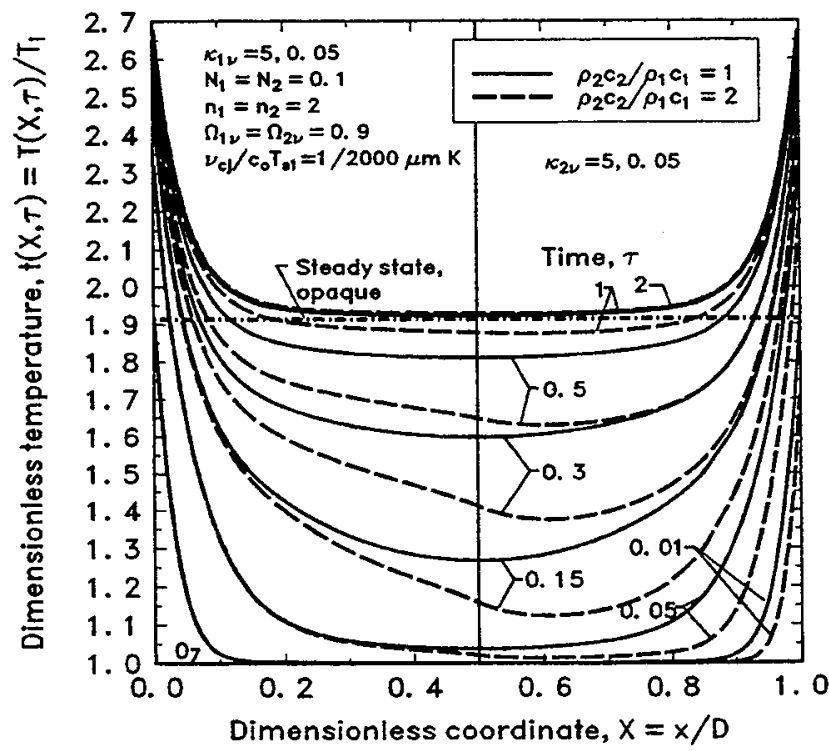

Fig. 2 Verification of two-layer analysis with scattering, and transient effect of increased heat capacity in the second layer; parameters: $\tilde{q}_{r 1}=\tilde{q}_{r 2}=1.0^{4}, H_{1}=H_{2}=5, t_{s 1}=t_{s 2}=4, \delta=0.5$, and $c_{21}=1$. 


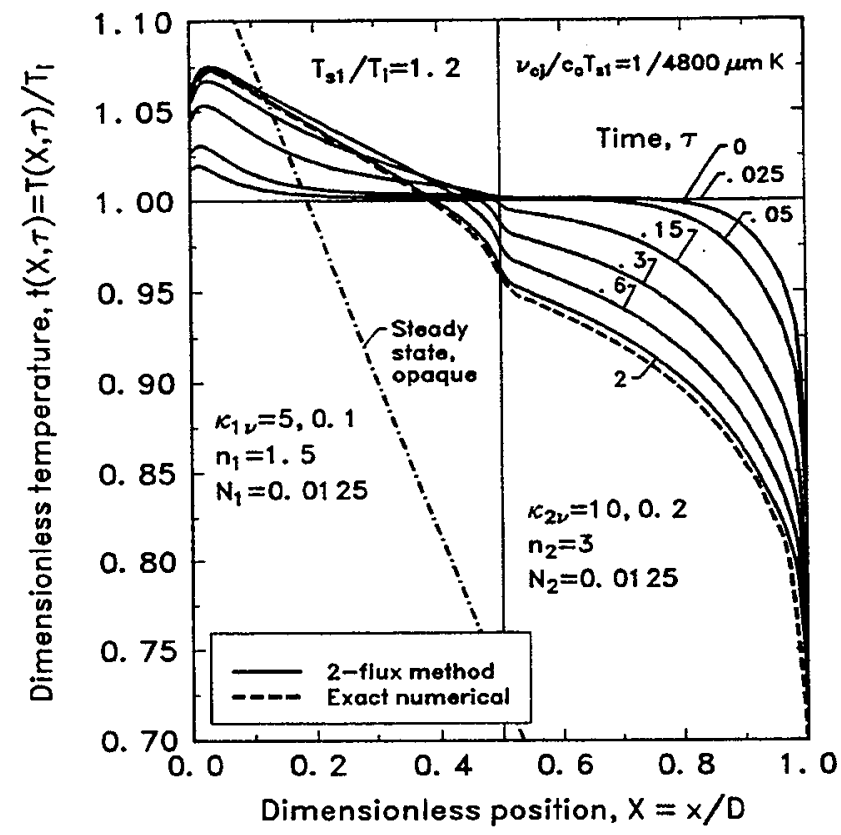

Fig. 3 Comparison of two-layer, two-band transient results at large time, with steady-state exact numerical results for radiative heating on one side; parameters: $\Omega_{1 \nu}=\Omega_{2 \nu}=0, \tilde{q}_{r 1}=1.2^{4}, \tilde{q}_{r 2}=$ $0.25^{4}, H_{1}=H_{2}=1, t_{g 1}=1, t_{g 2}=0.25, \delta=0.5$, and $c_{21}=1$.

posite with each layer having two spectral bands. For these results, the composite is heated by blackbody radiation at $X=$ 0 with temperature $T_{s 1}=1.2 T_{i}$. As the surface temperature rises at $X=0$, convective cooling occurs, since the gas temperature remains at the initial temperature $T_{g 1}=T_{i}$. There is stronger cooling by both radiation and convection at $X=1$, where there are lower blackbody surroundings and gas temperatures, $T_{s 2}=$ $T_{s 2}=0.25 T_{i}$. For comparison, the steady-state solution is shown (dot-dashed line) for a composite that is opaque, and so radiation exchange occurs only at the external surfaces, and there is only heat conduction within the interior. The transient solution has large internal radiation transfer and, at steady state, is considerably different from the opaque result.

After the solution method was verified in Figs. 2 and 3, and by some additional comparisons, it was used to examine a few transient effects to illustrate how the solution can be applied; these results are shown in Figs. 4-7. A few steady-state solutions are also shown for an opaque composite, and it is evident that in these examples, considerable radiation effects are present.

In Fig. 4 , the effect of adding scattering to the first layer is illustrated as a possible method to protect the second layer from external radiant heating. For the solid lines, the two-band spectral optical thicknesses of the first layer in order of increasing frequency are $\kappa_{1 \nu}=2$ and 0.1 . For the dashed lines, a uniform amount of scattering has been added to both bands so $\kappa_{1 \nu}=6.9$ and 5. The first layer has thus been made more opaque, and it tends to shield the second layer from the radiation incident at $X=0$; this significantly reduces the temperatures in the second layer. During the transient, however, the maximum temperature is increased in the first layer. At steady state, the dashed results approach somewhat more the temperature distribution for an opaque composite.

To illustrate the importance of spectral effects, the results without scattering in Fig. 4 are compared in Fig. 5 with temperatures when each layer is gray. In each layer the larger of the two-band optical thicknesses in Fig. 4 was used, and so, in Fig. $5, \kappa_{1 \nu}=2$ and $\kappa_{2 \nu}=5$ for all $\nu$. The bands have been eliminated that are more transparent, so that radiation incident

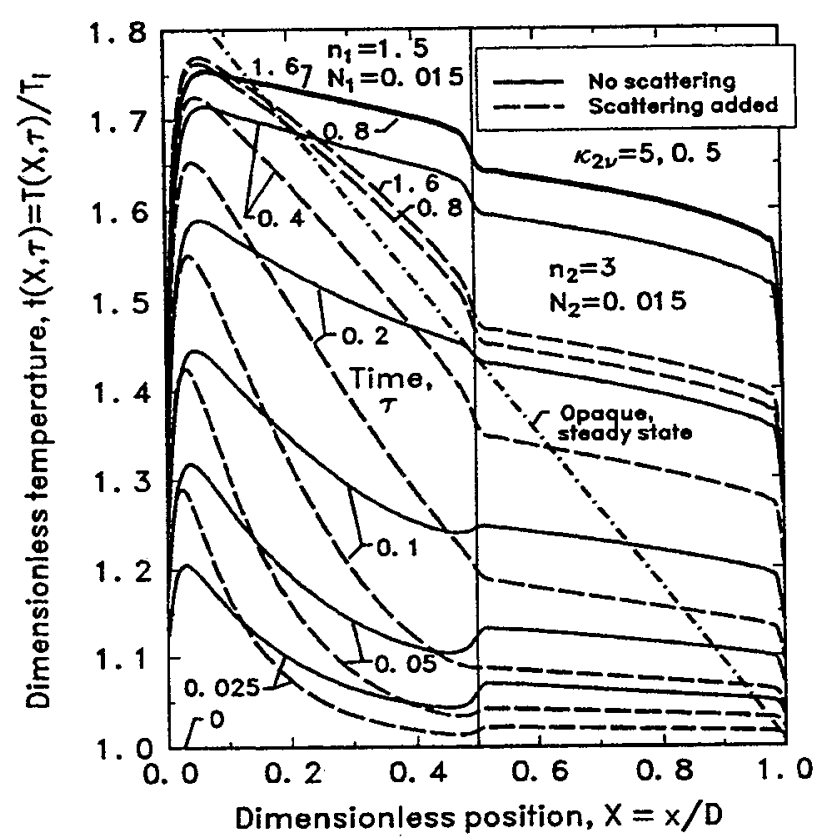

Fig. 4 Influence of scattering in first layer on transient temperatures after exposure to radiative heating on one side; parameters: $\tilde{q}_{r 1}=2^{4}, \tilde{q}_{r 2}=1^{4}, H_{1}=H_{2}=4, t_{g 1}=t_{g 2}=1, \delta=0.5, c_{21}=1$, and $\lambda_{c j} T_{s 1}=4000 \mu \mathrm{m} K$; solid lines, $\Omega_{1 v}=\Omega_{2 \nu}=0$ with $\kappa_{i \nu}=2$ and 0.1 ; dashed lines, $\Omega_{1 \nu}=0.7101$ and $\Omega_{2 \nu}=0.98$ with $\kappa_{1 \nu}=6.9$ and 5.

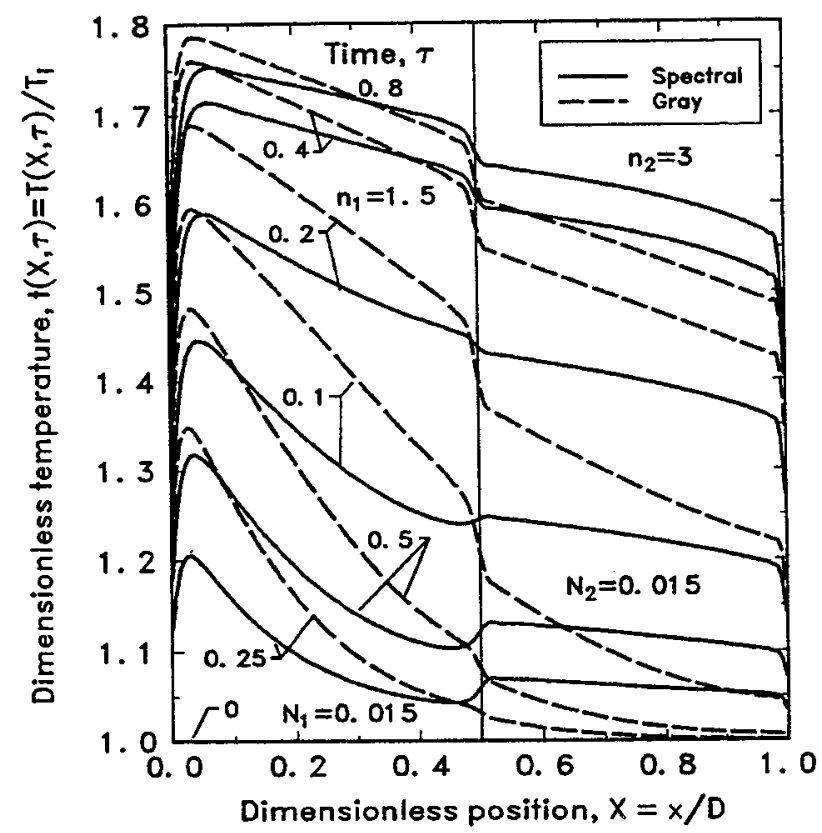

Fig. 5 Comparison of two-band spectral results with those for a gray composite; parameters: $\Omega_{1 \nu}=\Omega_{2 v}=0, \bar{q}_{r 1}=2^{4}, \bar{q}_{r 2}=1^{4}, H_{1}$ $=H_{2}=4, t_{g 1}=t_{g 2}=1, \delta=0.5$, and $c_{21}=1$; solid lines, $\kappa_{1 \nu}=2$ and $0.1, \kappa_{2 \nu}=5$ and 0.5 , and $\lambda_{c j} T_{s 1}=4000 \mu \mathrm{m} \mathrm{K}$; dashed lines, $\kappa_{1 \nu}=$ 2 and $\kappa_{2 \nu}=5$.

at $X=0$ is not transmitted as readily into the composite. Incident radiation is absorbed strongly in the region at small $X$. The result is increased maximum temperatures that are closer to $X=0$, and reduced temperatures in the second layer. By comparison with the dot-dashed line in Fig. 4 it is evident that the optical thicknesses in Fig. 5 are significantly smaller 


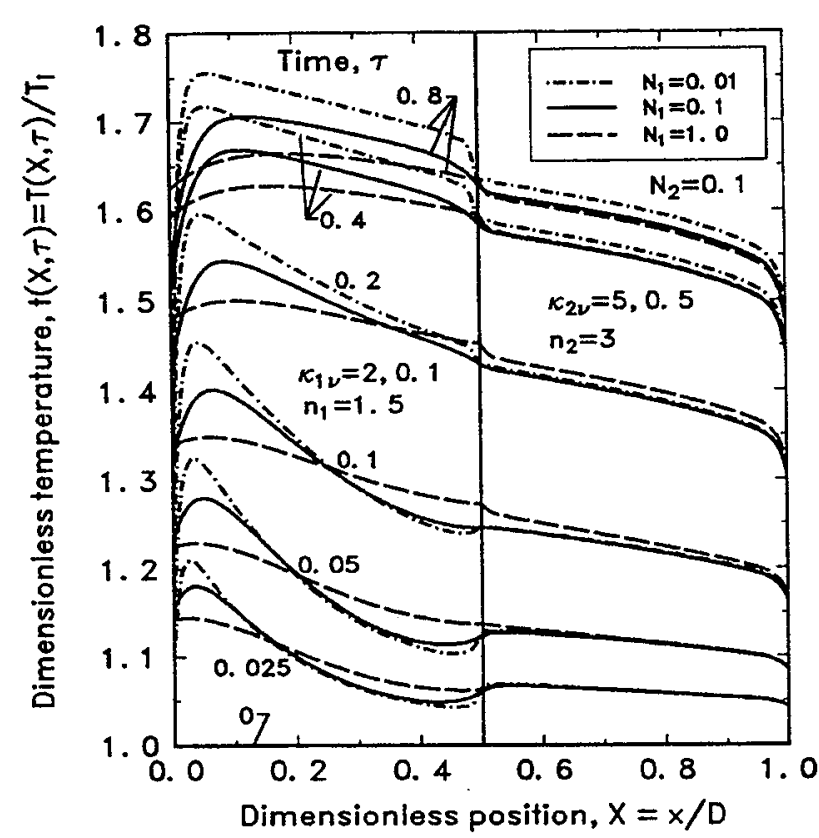

Fig. 6 Effect of thermal conductivity in the first layer on transient temperature distributions; parameters: $\mathbf{\Omega}_{1 \nu}=\mathbf{\Omega}_{2 \nu}=0, \tilde{q}_{r 1}=$ $2^{4}, \tilde{q}_{r 2}=1^{4}, H_{1}=H_{2}=4, t_{g 1}=t_{g 2}=1, \delta=0.5, c_{21}=1$, and $\lambda_{c j} T_{s 1}=$ $4000 \mu \mathrm{m} \mathrm{K}$.

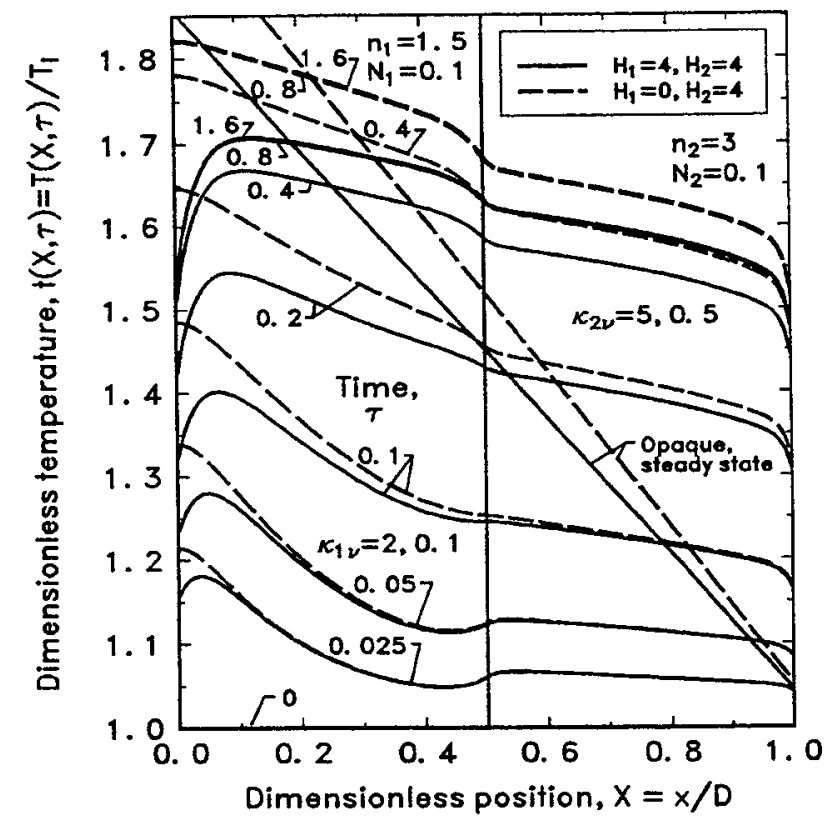

Fig. 7 Transient temperatures after exposure to radiation on one side without convective cooling; parameters: $\Omega_{1 v}=\Omega_{2 \nu}=\mathbf{0}, \tilde{q}_{r 1}=$ $2^{4}, \tilde{q}_{r 2}=1^{4}, t_{g 1}=t_{R 2}=1, \delta=0.5, c_{21}=1$, and $\lambda_{c j} T_{s 2}=4000 \mu \mathrm{m} \mathrm{K}$.

than those required for the composite to behave like an opaque material.

The results in Fig. 6 illustrate the effect of heat conduction in the first layer, which is the layer adjacent to the radiant heat addition. Except for the $N_{j}$ values, the parameters are the same as for the solid lines in Fig. 4. For the solid lines, $N_{1}=N_{2}=$ 0.1 , as compared with $N_{1}=N_{2}=0.015$ in Fig. 4 . The increased $N_{j}$ have a substantial effect in reducing the temperature gradients near the external boundaries, and the transient distributions are more uniform as expected. The dashed and dot- dashed curves show the effect of increasing and decreasing the conductivity of the first layer. An increase to $N_{1}=1$ removes practically all of the temperature peak near $X=0$. Decreasing the conductivity to $N_{1}=0.01$ produces a substantial temperature peak in the first layer and a large gradient near $X=0$ throughout the transient.

As a final illustrative example, Fig. 7 examines the effect of removing the convective cooling at the $X=0$ boundary. The solid lines are the same as the solid lines in Fig. 6, where $H_{1}$ $=4$; for the dashed lines, $H_{1}=0$. As expected, removing convective cooling at $X=0$ raises the temperatures near this boundary throughout the transient, and at $X=0$ the temperature distributions have a zero derivative. At steady state, the entire temperature profile is raised because of the reduced cooling on one side. The straight lines for an opaque composite at steady state with a uniform thermal conductivity illustrate, as in Fig. 4, that there are substantial internal radiation effects.

\section{Conclusions}

A combined analytical and numerical method was developed to obtain transient temperatures in a composite of two semitransparent layers with spectral radiation properties and with isotropic scattering. A Green's function solution was derived for the spectral two-flux equations in the composite. Summing over all frequencies provides the local radiative heat source. This was combined with an implicit numerical solution of the transient energy equation. The analysis accounts for heat conduction, and internal radiant absorption, emission, and isotropic scattering. An advantage of the method is that isotropic scattering is included without additional complexity. Starting from an initial temperature distribution, the layer is heated or cooled by external radiation and convection. The resulting steady-state and transient temperature distributions were verified by comparing with results where the radiative energy source was numerically evaluated from the exact spectral radiative transfer equations. Illustrative examples demonstrate how the method can be used to examine various heat transfer effects, and they show the comparison of transient spectral and gray calculations. An example is shown where the addition of scattering to a layer is used to shield a second layer from sudden exposure to radiative heating. The effects of changing the heat conductivity in part of the composite and removing the convective cooling at one boundary are also illustrated.

\section{References}

'Lii, C. C., and Özişik, M. N., "Transient Radiation and Conduction in an Absorbing, Emitting. Scattering Slab with Reflective Boundaries," International Journal of Heat and Mass Transfer, Vol. 15, No. 5, 1972, pp. 1175-1179.

${ }^{2}$ Wu, C.-Y., and Ou, N.-R., "Transient Two-Dimensional Radiative and Conductive Heat Transfer in a Scattering Medium," International Journal of Heat and Mass Transfer, Vol. 37, No. 17, 1994, pp. $2675-2686$.

${ }^{3}$ Siegel, R., “Two-Flux and Green's Function Method for Transient Radiative Transfer in a Semitransparent Layer," Proceedings of the Ist International Symposium on Radiative Transfer (Kuşadasi, Turkey), Radiative Transfer-I, Begell House, New York, 1996, pp. $473-487$

${ }^{4}$ Siegel, R., "Transient Heat Transfer in a Semitransparent Radiating Layer with Boundary Convection and Surface Reflections," International Journal of Heat and Mass Transfer, Vol. 39, No. 1, 1996. pp. $69-79$.

${ }^{5}$ Frankel, J. I., "Cumulative Variable Formulation for Transient Conductive and Radiative Transport in Participating Media," Journal of Thermophysics and Heat Transfer, Vol. 9, No. 2, 1995, pp. 210218.

${ }^{6}$ Field, R. E., and Viskanta, R., "Measurement and Prediction of Dynamic Temperatures in Unsymmetrically Cooled Glass Windows," Journal of Thermophysics and Heat Transfer, Vol. 7, No. 4, 1993, pp. 616-623.

${ }^{7}$ Tsai, J.-H., and Lin, J.-D., "Transient Combined Conduction and Radiation with Anisotropic Scattering," Journal of Thermophysics and Heat Transfer, Vol. 4, No. 1, 1990, pp. 92-97.

${ }^{8}$ Tsai, C.-F., and Nixon, G., "Transient Temperature Distribution of 
a Multilayer Composite Wall with Effects of Internal Thermal Radiation and Conduction," Numerical Heat Transfer, Vol. 10, No. 1, 1986, pp. 95-101.

'Timoshenko, V. P., and Trenev, M. G., "A Method for Evaluating Heat Transfer in Multilayer Semitransparent Materials," Heat Transfer-Soviet Research, Vol. 18, No. 5, 1986, pp. 44-57.

${ }^{10} \mathrm{Ho}$, C.-H., and Özisik, M. N., "Combined Conduction and Radiation in a Two-Layer Planar Medium with Flux Boundary Condition," Numerical Heat Transfer, Vol. 11, No. 3, 1987, pp. 321-340.

${ }^{11}$ Siegel, R., "Green's Function and Two-Flux Analysis for Transient Radiative Transfer in a Composite Layer," Proceedings of the American Society of Mechanical Engineers National Heat Transfer Conference (Houston, TX), Vol. 3, HTD-Vol. 325, 1996, pp. 35-43.

${ }^{12}$ Siddall, R. G., "Flux Methods for the Analysis of Radiant Heat
Transfer," Proceedings of the 4th Symposium on Flames and Industry, The Inst. of Fuel, Paper 16, 1972, pp. 169-177.

${ }^{13}$ Siegel, R., and Spuckler, C. M., "Approximate Solution Methods for Spectral Radiative Transfer in High Refractive Index Layers," International Journal of Heat and Mass Transfer, Vol. 37, Suppl. 1, 1994 , pp. $403-413$.

${ }^{14}$ Spuckler, C. M., and Siegel, R., "Two-Flux and Diffusion Methods for Radiative Transfer in Composite Layers,' Journal of Heat Transfer, Vol. 118, No. 1, 1996, pp. 218-222.

${ }^{15}$ Hildebrand, F. B., Methods of Applied Mathematics, PrenticeHall, New York, 1952, pp. 388, 389.

${ }^{16}$ Press, W. H., Flannery, B. P., Teukolsky, S. A., and Vetterling, W. T., Numerical Recipes, Cambridge Univ. Press, New York, 1989, pp. $40,41$. 